\title{
Climate change and health in North America: literature review protocol
}

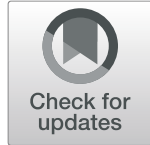

\author{
Sherilee L. Harper ${ }^{1 *} \mathbb{D}$, Ashlee Cunsolo², Amreen Babujee ${ }^{1}$, Shaugn Coggins ${ }^{1}$, Mauricio Domínguez Aguilar ${ }^{3}$ and \\ Carlee J. Wright ${ }^{1}$
}

\begin{abstract}
Background: Climate change is a defining issue and grand challenge for the health sector in North America. Synthesizing evidence on climate change impacts, climate-health adaptation, and climate-health mitigation is crucial for health practitioners and decision-makers to effectively understand, prepare for, and respond to climate change impacts on human health. This protocol paper outlines our process to systematically conduct a literature review to investigate the climate-health evidence base in North America.
\end{abstract}

Methods: A search string will be used to search CINAHL ${ }^{\oplus}$, Web of Science ${ }^{\top M}$, Scopus ${ }^{\oplus}$, Embase ${ }^{\oplus}$ via Ovid, and MEDL $I^{N E^{\oplus}}$ via Ovid aggregator databases. Articles will be screened using inclusion/exclusion criteria by two independent reviewers. First, the inclusion/exclusion criteria will be applied to article titles and abstracts, and then to the full articles. Included articles will be analyzed using quantitative and qualitative methods.

Discussion: This protocol describes review methods that will be used to systematically and transparently create a database of articles published in academic journals that examine climate-health in North America.

Keywords: Climate Change, Human Health, Mental Health, North America, Canada, United States of America, Mexico, Protocol

\section{Background}

The direct and indirect impacts of climate change on human health continue to be observed globally, and these wide-ranging impacts are projected to continue to increase and intensify this century $[1,2]$. The direct climate change effects on health include rising temperatures, which increase heat-related mortality and morbidity [3-5], and increased frequency and intensity of storms, resulting in increased injury, death, and psychological stressors [2, 6-8]. Indirect climate change impacts on health occur via altered environmental conditions, such as climate change impacts on water quality and quantity, which increase waterborne disease [9-13]; shifting ecosystems, which increase the risk of

* Correspondence: sherilee.harper@ualberta.ca

${ }^{1}$ School of Public Health, University of Alberta, ECHA, 1140587 Ave NW, Edmonton, AB T6G 1C9, Canada

Full list of author information is available at the end of the article foodborne disease [14-16], exacerbate food and nutritional security $[17,18]$, and change the range and distribution of vectors that cause vectorborne disease $[19,20]$; and place-based connections and identities, leading to psycho-social stressors and potential increases in negative mental health outcomes and suicide $[6,8]$. These wide-ranging impacts are not uniformly or equitably distributed: children, the elderly, those with pre-existing health conditions, those experiencing lower socioeconomic conditions, women, and those with close connections to and reliance upon the local environment (e.g. Indigenous Peoples, farmers, fishers) often experience higher burdens of climate-health impacts $[1,2,21]$. Indeed, climate change impacts on human health not only are dependent on exposure to climatic and environmental changes, but also depend on climate change sensitivity and adaptive capacity-both of which are 
underpinned by the social determinants of health $[1,22$, 23].

The inherent complexity, great magnitude, and widespread, inequitable, and intersectional distribution of climate change impacts on health present an urgent and grand challenge for the health sector this century [2, 24, 25]. Climate-health research and evidence is critical for informing effective, equitable, and timely adaptation responses and strategies. For instance, research continues to inform local to international climate change and health vulnerability and adaptation assessments [26]. However, to create evidence-based climate-health adaptation strategies, health practitioners, researchers, and policy makers must sift and sort through vast and often unmanageable amounts of information. Indeed, the global climate-health evidence base has seen exponential growth in recent years, with tens of thousands of articles published globally this century [22, 25, 27, 28]. Even when resources are available to parse through the evidence base, the available research evidence may not be locally pertinent to decision-makers, may provide poor quality of evidence, may exclude factors important to decision-makers, may overlook temporal and geographical scales over which decision-makers have impact, and/ or may not produce information in a timely manner [29-37].

Literature reviews that utilize systematic methods present a tool to efficiently and effectively integrate climate-health information and provide data to support evidence-based decision-making. Furthermore, literature reviews that use systematic methods are replicable and transparent, reduce bias, and are ultimately intended to improve reliability and accuracy of conclusions. As such, systematic approaches to identify, explore, evaluate, and synthesize literature separates insignificant, less rigorous, or redundant literature from the critical and noteworthy studies that are worthy of exploration and consideration [38]. As such, a systematic approach to synthesizing the climate-health literature provides invaluable information and adds value to the climate-health evidence base from which decision-makers can draw from. Therefore, we aim to systematically and transparently create a database of articles published in academic journals that examine climate-health in North America. As such, we outline our protocol that will be used to systematically identify and characterize literature at the climate-health nexus in North America.

\section{Methods}

This protocol was designed in accordance with the Preferred Reporting Items for Systematic Review and MetaAnalyses (PRISMA) Guidelines [39, 40] and presented in accordance with the PRISMA-P checklist.

\section{Research questions}

Research on climate change and human health encompasses a diverse range of health outcomes, climate change exposures, populations, and study designs. Given the breadth and depth of information needed by health practitioners and decision-makers, a variety of research questions will be examined (Table 1).

\section{Search strategy}

The search strategy, including the search string development and selection of databases, was developed in consultation with a research librarian and members of the research team (SLH, AC, and MDA). The search string contains terms related to climate change [41, 42], human health outcomes $[1,25,43,44]$, and study location (Table 2). Given the interdisciplinary nature of the climate-health nexus and to ensure that our search is comprehensive, the search string will be used to search five academic databases:

$1 \mathrm{CINAHL}^{\circ}$ will be searched to capture unique literature not found in other databases on common disease and injury conditions, as well as other health topics;

2 Web of Science ${ }^{\mathrm{Tw}}$ will be searched to capture a wide range of multi-disciplinary literature;

3 Scopus $^{\bullet}$ will be searched to capture literature related to medicine, technology, science, and social sciences;

4 Embase $^{\bullet}$ via Ovid will be searched to capture a vast range of biomedical sciences journals; and

5 MEDLINE $^{\ominus}$ via Ovid will be searched to capture literature on biomedical and health sciences.

No language restrictions will be placed on the search. Date restrictions will be applied to capture literature published on or after 01 January 2013, in order to capture literature published after the Intergovernmental Panel on Climate Change (IPCC) Fifth Assessment Report (which assessed literature accepted for publication prior to 31 August 2013). An initial test search was conducted on June 10, 2019, and updated on February 14, 2020; however, the search will be updated to include literature published within the most recent full calendar year prior to publication.

To explore the sensitivity of our search and capture any missed articles, (1) a snowball search will be conducted on the reference lists of all the literature that meet the inclusion criteria and (2) a hand search of three relevant disciplinary journals will be conducted:

1 Environmental Health Perspectives, an open access peer-reviewed journal that is a leading disciplinary journal within environmental health sciences; 
Table 1 Examples of research questions guiding the climate change and health in North America literature review

\section{Example questions}

1. What types of published literature exist on climate change and health in North America?

- Primary research articles

- Review articles using systematic methods

2. What are the North American publication trends over time?

- Year of article publication

3. What is the geographical distribution of research and data in North America? • Canada

- United States of America (USA)

- Mexico

- Multinational, including North America

4. What types of climate-health data are being assessed in the literature? What is the nature of climate-health literature and state-of-knowledge for different health outcomes?

- Weather data (e.g. temperature, UV, precipitation)

- Climatic hazard data (e.g. hurricanes, wildfires, heat events, air quality)

- Health data (e.g. heat morbidity and/or mortality, respiratory illness, vectorborne diseases)

- Social characteristics as they relate to impacts on climatesensitive health outcomes (e.g. gender, income, education, ethnicity)

5. What aspects of climate change are being focused on in North America?

- Climate change impacts

- Climate change adaptation

- Climate change mitigation

6. What research methodologies are being used?

- Qualitative research/quantitative research/mixed qualitative and quantitative research

- Inclusion of future climate projections

7. What are key climate-health risks in North America?

- Intersection of climatic variables, health outcomes, and social characteristics

8. What climate-health adaptation strategies are effective in North America, for - Climate change adaptation whom, under what conditions, and why?

2 The Lancet, a peer-reviewed journal that is the leading disciplinary journal within public health sciences; and

3 Climatic Change, a peer-reviewed journal covering cross-disciplinary literature that is a leading disciplinary journal for climate change research.
Citations will be downloaded from the databases and uploaded into Mendeley ${ }^{\mathrm{Tm}}$ reference management software to facilitate reference management, article retrieval, and removal of duplicate citations. Then, de-duplicated citations will be uploaded into DistillerSR ${ }^{\circ}$ to facilitate screening.

Table 2 Search strategy for $\mathrm{CINAHL}^{\oplus}$ to identify published articles reporting on climate change impacts on human health in North America published after 2013 (see Appendix 1 for search strategies for Web of Science ${ }^{\mathrm{TM}}$, Scopus ${ }^{\oplus}$, Embase ${ }^{\oplus}$ via Ovid, and MEDLINE ${ }^{\circledast}$ via Ovid)

\begin{tabular}{|c|c|}
\hline Component & Search term \\
\hline $\begin{array}{l}\text { North } \\
\text { America }\end{array}$ & $\begin{array}{l}\text { Canada OR "North America" OR "United States" OR "United States of America" OR USA OR Mexico OR "United Mexican States" OR } \\
\text { "British Columbia" OR Alberta OR Manitoba OR Saskatchewan OR Ontario OR Quebec OR "Prince Edward Island" OR PEl OR "Nova } \\
\text { Scotia" OR "New Brunswick" OR Newfoundland OR Labrador OR Yukon OR Nunavut OR "Northwest Territories" OR NWT OR Alabama } \\
\text { OR Alaska OR Arizona OR Arkansas OR California OR Colorado OR Connecticut OR Delaware OR Florida OR Georgia OR Hawaii OR } \\
\text { Idaho OR Illinois OR Indiana OR lowa OR Kansas OR Kentucky OR Louisiana OR Maine OR Maryland OR Massachusetts OR Michigan } \\
\text { OR Minnesota OR Mississippi OR Missouri OR Montana OR Nebraska OR Nevada OR "New Hampshire" OR "New Jersey" OR "New } \\
\text { Mexico" OR "New York" OR "North Carolina" OR "North Dakota" OR Ohio OR Oklahoma OR Oregon OR Pennsylvania OR "Rhode } \\
\text { Island" OR "South Carolina" OR "South Dakota" OR Tennessee OR Texas OR Utah OR Vermont OR Virginia OR Washington OR "West } \\
\text { Virginia" OR Wisconsin OR Wyoming OR Aguascalientes OR "Baja California" OR Campeche OR Chiapas OR Chihuahua OR Coahuila OR } \\
\text { Colima OR Durango OR Guanajuato OR Guerrero OR Hidalgo OR Jalisco OR México OR Michoacán OR Morelos OR Nayarit OR "Nuevo } \\
\text { León" OR Oaxaca OR Puebla OR Querétaro OR "Quintana Roo" OR "San Luis Potosi" OR Sinaloa OR Sonora OR Tabasco OR Tamaulipas } \\
\text { OR Tlaxcala OR Veracruz OR Yucatán OR Zacatecas } \\
\text { AND }\end{array}$ \\
\hline $\begin{array}{l}\text { Climate } \\
\text { change }\end{array}$ & $\begin{array}{l}\text { "climate change" OR weather OR "atmospheric pressure" OR "climatic change" OR "global warming" OR "environmental change" OR } \\
\text { "climate disaster" OR "greenhouse effect" OR "climate variability" OR "climatic variability" OR "carbon emission" OR cold OR cool OR } \\
\text { cooling OR heat OR humid* OR ice OR precipitation OR rain* OR season* OR snow* OR storm OR temperature OR warm OR warming } \\
\text { OR wind OR "ultraviolet radiation" OR UV } \\
\text { AND }\end{array}$ \\
\hline $\begin{array}{l}\text { Human } \\
\text { health }\end{array}$ & $\begin{array}{l}\text { health OR disease* OR morbidity OR mortality OR wellbeing OR illness* OR wellness OR infect* OR death OR injur* OR mental* OR } \\
\text { emotion* }\end{array}$ \\
\hline
\end{tabular}




\section{Article selection Inclusion and exclusion criteria}

To be included, articles must evaluate or examine the intersection of climate change and human health in North America (Fig. 1). Health is defined to include physical, mental, emotional, and social health and wellness [1, 25, 43, 44] (Fig. 1). This broad definition will be used to examine the nuanced and complex direct and indirect impacts of climate change on human health. To examine the depth and breadth of climate change impacts on health, climate change contexts are defined to include seasonality, weather parameters, extreme weather events, climate, climate change, climate variability, and climate hazards [41, 42] (Fig. 1). However, articles that discuss climate in terms of indoor work environments, non-climate hazards due to geologic events (e.g. earthquakes), and non-anthropogenic climate change (e.g. due to volcanic eruptions) will be excluded. This broad definition of climate change contexts will be used in order to examine the wide range and complexity of climate change impacts on human health. To be included, articles need to explicitly link health outcomes to climate change in the goal statement, methods section, and/or results section of the article. Therefore, articles that discuss both human health and climate change-but do not link the two together-will be excluded. The climate-health research has to take place in
North America to be included. North America is defined to include Canada, the USA, and Mexico in order to be consistent with the IPCC geographical classifications; that is, in the Fifth Assessment Report, the IPCC began confining North America to include Canada, Mexico, and the USA [45] (Fig. 1). Articles published in any language will be eligible for inclusion. Articles need to be published online on or after 01 January 2013 to be included. No restrictions will be placed on population type (i.e. all human studies will be eligible for inclusion).

\section{Level 1 screening}

The title and abstract of each citation will be examined for relevance. A stacked questionnaire will be used to screen the titles and abstracts; that is, when a criterion is not met, the subsequent criteria will not be assessed. When all inclusion criteria are met and/or it is unclear whether or not an inclusion criterion is met (e.g. "unsure"), the article will proceed to Level 2 screening. If the article meets any exclusion criteria, it will not proceed to Level 2 screening. Level 1 screening will be completed by two independent reviewers, who will meet to resolve any conflicts via discussion. The level of agreement between reviewers will be evaluated by dividing the total number of conflicts by the total number of articles screened for Level 1.

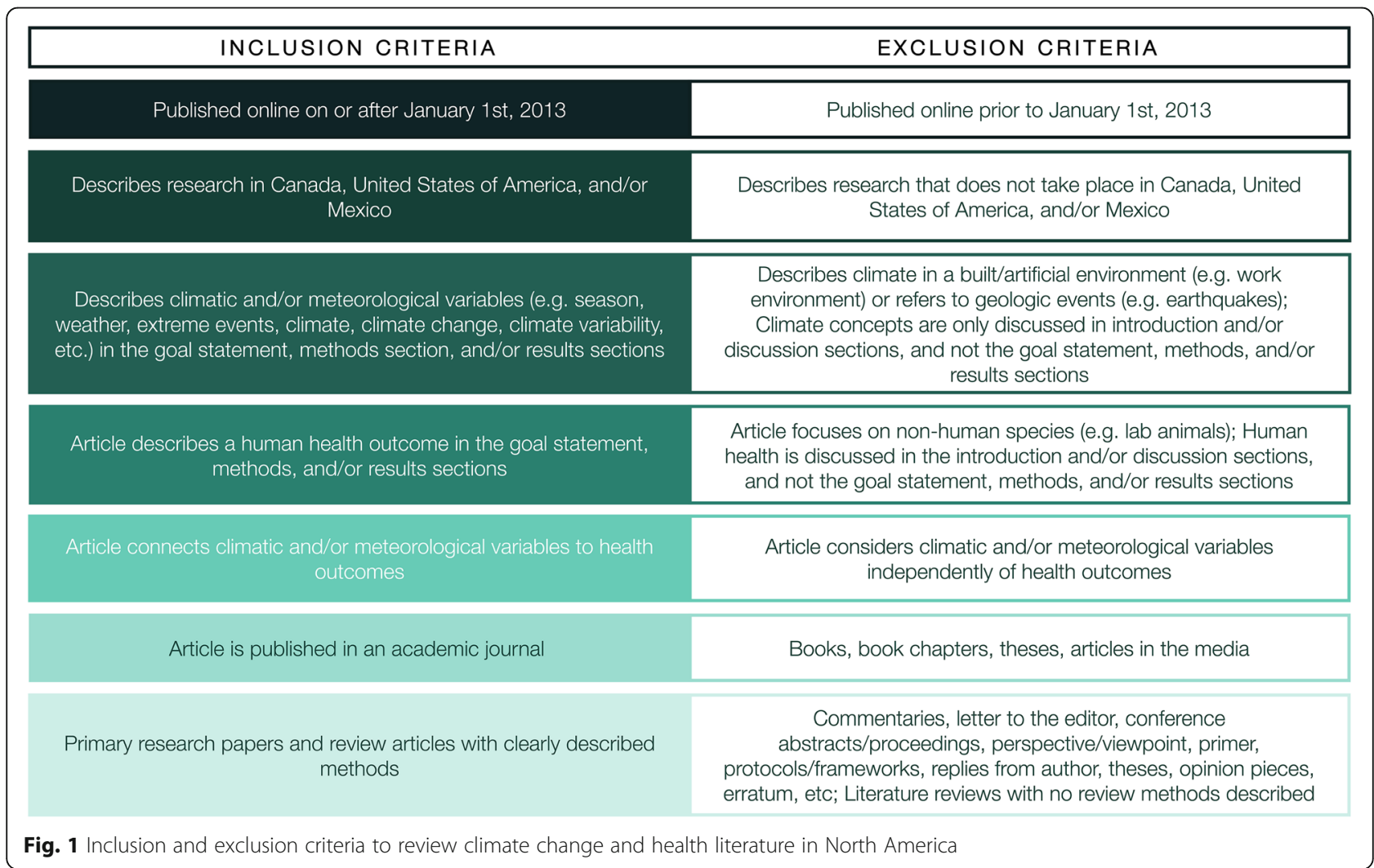

Fig. 1 Inclusion and exclusion criteria to review climate change and health literature in North America 


\section{Level 2 screening}

The full text of all potentially relevant articles will be screened for relevance. A stacked questionnaire will also be used to screen the full texts. In Level 2 screening, only articles that meet all the inclusion criteria will be included in the review (i.e. "unsure" will not be an option). Level 2 screening will be completed by two independent reviewers, who will meet to resolve any conflicts via discussion. The level of agreement between reviewers will be evaluated by dividing the total number of conflicts by the total number of articles screened for Level 2 (Fig. 2).

\section{Data extraction and analysis}

A data extraction form will be created in DistillerSR ${ }^{\odot}$ (Appendix 2) and will be tested by three data extractors on a sample of articles to allow for calibration on the extraction process (i.e. $5 \%$ of articles if greater than 50 articles, $10 \%$ of articles if less than or equal to 50 articles). After completing the calibration process, the form will be adapted based on feedback from the extractors to improve usability and accuracy. The data extractors will then use the data extraction form to complete data extraction. Reviewers will meet regularly to discuss and resolve any further issues in data extraction, in order to ensure the data extraction process remains consistent across reviewers.

Data will be extracted from original research papers (i.e. articles containing data collection and analysis) and review articles that reported a systematic methodology. This data extraction will focus on study characteristics, including the country that the data were collected in, focus of the study (i.e. climate change impact, adaptation, and/or mitigation), weather variables, climatic hazards, health outcomes, social characteristics, and future projections. The categories within each study characteristic will not be mutually exclusive, allowing more than one response/category to be selected under each study characteristic. For the country of study, Canada, the USA, and/or Mexico will be selected if the article describes data collection in each country respectively. Non-North American regions will be selected if the article not only collects data external to North America, but also includes data collection within Canada, the USA, and/or Mexico. For the study focus, data will be extracted on whether the article focuses on climate change impacts, adaptation, and/or mitigation within the goals, methods, and/or results sections of the article. Temperature, precipitation, and/or UV radiation will be selected for weather variables if the article utilizes these data in the goal, methods, and/or results sections. Data will be extracted on the following climatic hazards if the article addresses them in the goal, methods, and/or results sections: heat events (e.g. extreme heat, heat waves), cold events (e.g. extreme cold, winter storms), air quality (e.g. pollution, parts per million (PPM) data, greenhouse gas emissions), droughts, flooding, wildfires, hurricanes, wildlife changes (including changes in disease vectors such as ticks or mosquitos), vegetation changes (including changes in pollen), freshwater (including drinking water), ocean conditions (including sea level rise and ocean acidity/salinity/temperature changes), ice extent/stability/duration (including sea ice and freshwater ice), coastal erosion, permafrost changes, and/or environmental hazards (e.g. exposure to sewage, reduced crop productivity).

Data will be extracted on the following health outcomes if the article focuses on them within the goal, methods, and/or results sections: heat-related morbidity and/or mortality, respiratory outcomes (including asthma, chronic obstructive pulmonary disease), cardiovascular outcomes (including heart attacks or stroke), urinary outcomes (e.g. urinary tract infections, renal failure), dermatologic concerns, mental health and wellness (e.g. suicide, emotional health), fetal health/birth outcomes and/or maternal health, cold exposure, allergies, nutrition (including nutrient deficiency), waterborne disease, foodborne disease, vectorborne disease, injuries (including accidents), and general morbidity and/or mortality. Data on the following social characteristics will also be extracted from the articles if they are included in the goal, methods, and/or results sections of the article: access to healthcare, sex and/or gender, age, income, livelihood (including data on employment, occupation), ethnicity, culture, Indigenous Peoples, rural/ remote communities ("rural", "remote", or similar terminology must be explicitly mentioned), urban communities ("urban", "city", "metropolitan", or similar terminology must be explicitly used), coastal communities (use of "coastal", or similar terms must be explicitly mentioned), residence location (zipcode/postal code, neighbourhood, etc.), level of education, and housing (e.g. data on size, age, number of windows, air conditioning). Finally, data will be collected on future projections, including projections that employ qualitative and/ or quantitative methods that are included in the goal, methods, and/or results sections of the article.

Descriptive statistics and regression modelling will be used to examine publication trends. Data will be visualized through the use of maps, graphs, and other visualization techniques as appropriate. To enable replicability and transparency, a PRISMA flowchart will be created to illustrate the article selection process and reasons for exclusion. Additionally, qualitative thematic analyses will be conducted. These analyses will utilize constant-comparative approaches to identify patterns across articles through the identification, development, and refinement of codes and themes. Article excerpts 


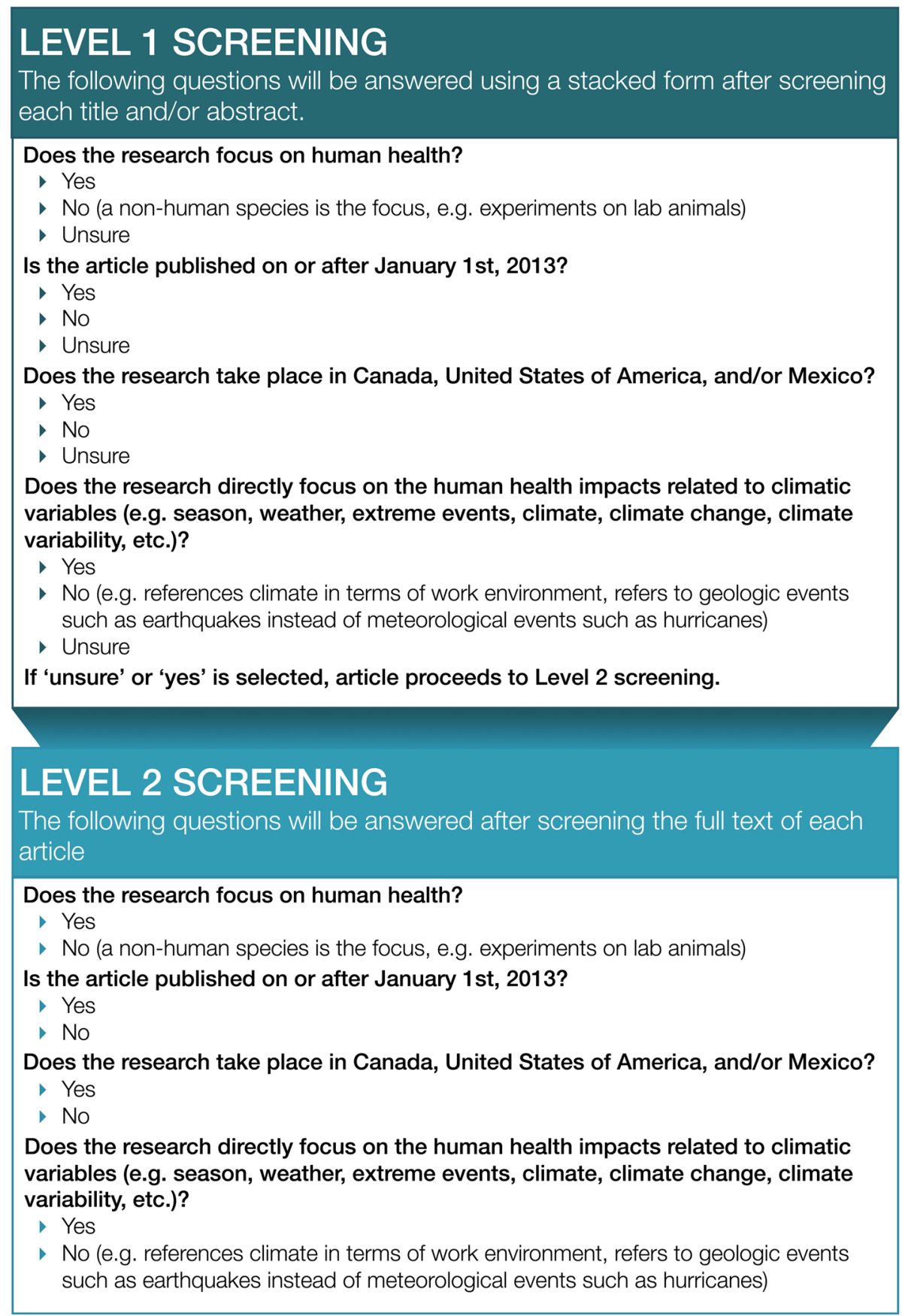

Fig. 2 Flow chart of screening questions for the literature review on climate change and health in North America

will be grouped under thematic categories in order to explore connections in article characteristics, methodologies, and findings.

Quality appraisal of studies included in the systematic scoping review will be performed using a framework based on the Mixed Methods Appraisal Tool (MMAT) [46] and the Confidence in the Evidence from Reviews of Qualitative Research (CERQual) tool [47]. This will enable appraisal of evidence in reviews that contain qualitative, quantitative, and mixed methods studies, as well as appraisal of methodological limitations in included qualitative studies. These tools may be adapted to include additional questions as required in order to fit the scope and objectives of the review. A minimum of two reviewers will independently appraise the included articles and discuss judgements as needed. The findings will be made available as supplementary material for the review. 


\section{Discussion}

Climate-health literature reviews using systematic methods will be increasingly critical in the health sector, given the depth and breadth of the growing body of climate change and health literature, as well as the urgent need for evidence to inform climate-health adaptation and mitigation strategies. To support and encourage the systematic and transparent identification and synthesis of climate-health information, this protocol describes our approach to systematically and transparently create a database of articles published in academic journals that examine climate-health in North America.

\section{Appendix 1}

Search strategy for CINAHL ${ }^{\oplus}$, Web of Science ${ }^{\mathrm{Tm}}$, Scopus ${ }^{\oplus}$, Embase $^{\oplus}$ via Ovid, and MEDLINE ${ }^{\oplus}$ via Ovid.

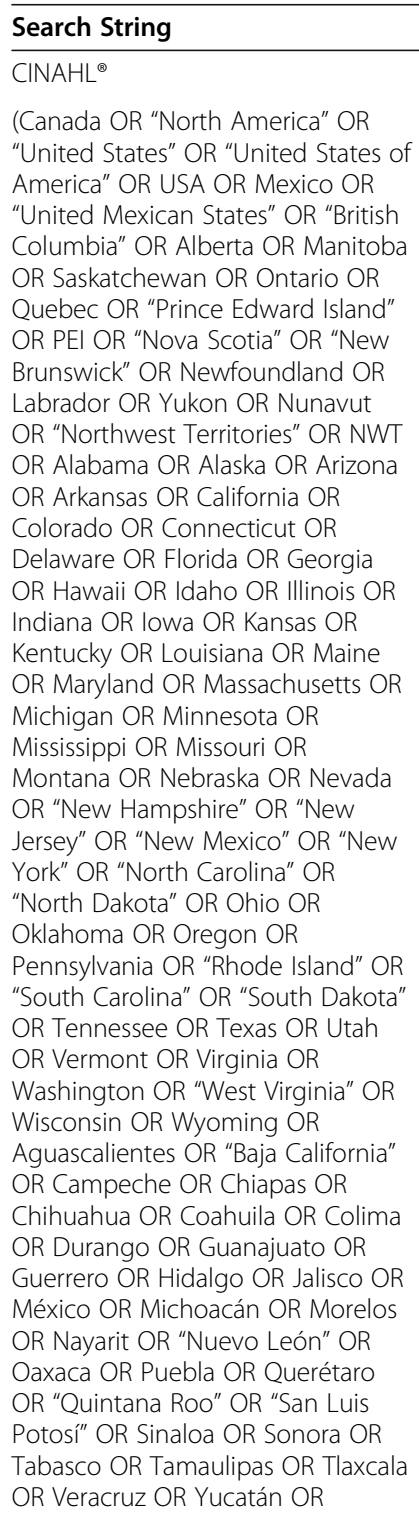

Appendix 1 (Continued)

Search String

Search Limiters

Zacatecas) AND ("climate change"

OR weather OR "atmospheric pressure" OR "climatic change" OR "global warming" OR

"environmental change" OR

"climate disaster" OR "greenhouse

effect" OR "climate variability" OR

"climatic variability" OR "carbon

emission" OR cold OR cool OR

cooling OR heat OR humid* OR ice

OR precipitation OR rain* OR

season* OR snow* OR storm OR

temperature OR warm OR warming

$\mathrm{OR}$ wind OR "ultraviolet radiation"

OR UV) AND (health OR disease*

OR morbidity OR mortality OR

wellbeing OR illness* OR wellness

$O R$ infect* $O R$ death $O R$ injur* $O R$

mental* OR emotion*)

Web of Science ${ }^{\text {TM }}$

TOPIC: (Canada OR "North America" OR "United States" OR "United States of America" OR USA OR

Mexico OR "United Mexican States"

OR "British Columbia" OR Alberta

OR Manitoba OR Saskatchewan OR

Ontario OR Quebec OR "Prince

Edward Island" OR PEI OR "Nova

Scotia" OR "New Brunswick" OR

Newfoundland OR Labrador OR

Yukon OR Nunavut OR "Northwest

Territories" OR NWT OR Alabama

OR Alaska OR Arizona OR Arkansas

OR California OR Colorado OR

Connecticut OR Delaware OR

Florida OR Georgia OR Hawaii OR

Idaho OR Illinois OR Indiana OR

lowa OR Kansas OR Kentucky OR

Louisiana OR Maine OR Maryland

OR Massachusetts OR Michigan OR

Minnesota OR Mississippi OR

Missouri OR Montana OR Nebraska

OR Nevada OR "New Hampshire"

OR "New Jersey" OR "New Mexico"

OR "New York" OR "North Carolina"

OR "North Dakota" OR Ohio OR

Oklahoma OR Oregon OR

Pennsylvania OR "Rhode Island" OR

"South Carolina" OR "South Dakota"

OR Tennessee OR Texas OR Utah

OR Vermont OR Virginia OR

Washington OR "West Virginia" OR

Wisconsin OR Wyoming OR

Aguascalientes OR "Baja California"

OR Campeche OR Chiapas OR

Chihuahua OR Coahuila OR Colima

OR Durango OR Guanajuato OR

Guerrero OR Hidalgo OR Jalisco OR

México OR Michoacán OR Morelos

OR Nayarit OR "Nuevo León" OR

Oaxaca OR Puebla OR Querétaro

OR "Quintana Roo" OR "San Luis

Potosi" OR Sinaloa OR Sonora OR

Tabasco OR Tamaulipas OR Tlaxcala

OR Veracruz OR Yucatán OR

Zacatecas) AND TOPIC: ("climate

change" OR weather OR
Publication date: 2013 2019Countries: USA, Canada, Mexico 
Appendix 1 (Continued)

Search String

"atmospheric pressure" OR "climatic

change" OR "global warming" OR

"environmental change" OR

"climate disaster" OR "greenhouse

effect" OR "climate variability" OR

"climatic variability" OR "carbon

emission" OR cold OR cool OR

cooling OR heat OR humid* OR ice

OR precipitation OR rain* OR

season* OR snow* OR storm OR

temperature OR warm OR warming

OR wind OR "ultraviolet radiation"

OR UV) AND TOPIC: (health OR

disease* OR morbidity OR mortality

OR wellbeing OR illness* OR

wellness OR infect* OR death OR

injur* OR mental* OR emotion ${ }^{*}$ )

Scopus ${ }^{\circledast}$

(TITLE-ABS-KEY (canada OR "North America" OR "United States" OR

"United States of America" OR usa

OR mexico OR "United Mexican

States" OR "British Columbia" OR

alberta OR manitoba OR

saskatchewan OR ontario OR

quebec OR "Prince Edward Island"

OR pei OR "Nova Scotia" OR "New

Brunswick" OR newfoundland OR

labrador OR yukon OR nunavut OR

"Northwest Territories" OR nwt OR

alabama OR alaska OR arizona OR

arkansas OR california OR colorado

OR connecticut OR delaware OR

florida OR georgia OR hawaii OR

idaho OR illinois OR indiana OR

iowa OR kansas OR kentucky OR

louisiana OR maine OR maryland

OR massachusetts OR michigan OR

minnesota OR mississippi OR

missouri OR montana OR nebraska

OR nevada OR "New Hampshire"

OR "New Jersey" OR "New Mexico"

OR "New York" OR "North Carolina"

OR "North Dakota" OR ohio OR

oklahoma OR oregon OR

pennsylvania OR "Rhode Island" OR

"South Carolina" OR "South Dakota"

OR tennessee OR texas OR utah OR vermont $O R$ virginia $O R$

washington OR "West Virginia" OR wisconsin OR wyoming OR

aguascalientes OR "Baja California"

OR campeche OR chiapas OR

chihuahua OR coahuila OR colima

OR durango OR guanajuato OR

guerrero OR hidalgo OR jalisco OR

méxico OR michoacán OR morelos

OR nayarit OR "Nuevo León" OR

oaxaca OR puebla OR querétaro OR

"Quintana Roo" OR "San Luis

Potosí" OR sinaloa OR sonora OR

tabasco OR tamaulipas OR tlaxcala

OR veracruz OR yucatán OR

zacatecas)) AND (TITLE-ABS-KEY

("climate change" OR weather OR

"atmospheric pressure" OR "climatic

change" OR "global warming" OR
Appendix 1 (Continued)

\section{Search String}

Search Limiters

Search Limiters

"environmental change" OR

"climate disaster" OR "greenhouse

effect" OR "climate variability" OR

"climatic variability" OR "carbon

emission" OR cold OR cool OR

cooling OR heat OR humid* OR ice

OR precipitation OR rain* OR

season* OR snow* OR storm OR

temperature OR warm OR warming

OR wind OR "ultraviolet radiation"

OR uv)) AND (TITLE-ABS-KEY (health

OR disease* OR morbidity OR

mortality OR wellbeing OR illness*

OR wellness OR infect* OR death

OR injur* OR mental* OR

emotion*)) AND (LIMIT-TO (AFFI

LCOUNTRY, "United States") OR

LIMIT-TO (AFFIL COUNTRY,

"Canada") OR LIMIT-TO (AFFI

LCOUNTRY, "Mexico")) AND (LIMIT-

TO (PUBYEAR, 2019) OR LIMIT-TO

(PUBYEAR, 2018) OR LIMIT-TO

(PUBYEAR, 2017) OR LIMIT-TO

(PUBYEAR, 2016) OR LIMIT-TO

(PUBYEAR, 2015) OR LIMIT-TO

(PUBYEAR, 2014) OR LIMIT-TO

(PUBYEAR, 2013)) AND (LIMIT-TO

(EXACTKEYWORD, "Human") OR

LIMIT-TO (EXACTKEYWORD,

"Humans"))

Embase $^{\circledast}$ via Ovid

(Canada or "North America" or

Publication date: 2013-

"United States" or "United States of

America" or USA or Mexico or

"United Mexican States" or "British

Columbia" or Alberta or Manitoba

or Saskatchewan or Ontario or

Quebec or "Prince Edward Island"

or PEl or "Nova Scotia" or "New

Brunswick" or Newfoundland or

Labrador or Yukon or Nunavut or

"Northwest Territories" or NWT or

Alabama or Alaska or Arizona or

Arkansas or California or Colorado

or Connecticut or Delaware or

Florida or Georgia or Hawaii or

Idaho or Illinois or Indiana or lowa

or Kansas or Kentucky or Louisiana

or Maine or Maryland or

Massachusetts or Michigan or

Minnesota or Mississippi or Missouri

or Montana or Nebraska or Nevada

or "New Hampshire" or "New

Jersey" or "New Mexico" or "New

York" or "North Carolina" or "North

Dakota" or Ohio or Oklahoma or

Oregon or Pennsylvania or "Rhode

Island" or "South Carolina" or

"South Dakota" or Tennessee or

Texas or Utah or Vermont or

Virginia or Washington or "West

Virginia" or Wisconsin or Wyoming

or Aguascalientes or "Baja

California" or Campeche or Chiapas

or Chihuahua or Coahuila or

Colima or Durango or Guanajuato

or Guerrero or Hidalgo or Jalisco or
2019Filtered by: Humans 
Appendix 1 (Continued)

Search String

Search Limiters

Mexico or Michoacan or Morelos or Nayarit or "Nuevo Leon" or Oaxaca or Puebla or Queretaro or "Quintana Roo" or "San Luis Potosi" or Sinaloa or Sonora or Tabasco or Tamaulipas or Tlaxcala or Veracruz or Yucatan or Zacatecas).mp. [mp = title, abstract, heading word, drug trade name, original title, device manufacturer, drug manufacturer, device trade name, keyword, floating subheading word, candidate term word] AND ("climate change" or weather or "atmospheric pressure" or "climatic change" or "global warming" or "environmental change" or "climate disaster" or "greenhouse effect" or "climate variability" or "climatic variability" or "carbon emission" or cold or cool or cooling or heat or humid* or ice or precipitation or rain* or season* or snow* or storm or temperature or warm or warming or wind or "ultraviolet radiation" or UV).mp. [mp = title, abstract, heading word, drug trade name, original title, device manufacturer, drug manufacturer, device trade name, keyword, floating subheading word, candidate term word] AND (health or disease* or morbidity or mortality or wellbeing or illness* or wellness or infect* or death or injur* or mental* or emotion*).mp. $[\mathrm{mp}=$ title, abstract, heading word, drug trade name, original title, device manufacturer, drug manufacturer, device trade name, keyword, floating subheading word, candidate term word]

\section{MEDLINE ${ }^{\circledR}$ via Ovid}

(Canada or "North America" or "United States" or "United States of America" or USA or Mexico or "United Mexican States" or "British Columbia" or Alberta or Manitoba or Saskatchewan or Ontario or Quebec or "Prince Edward Island" or PEl or "Nova Scotia" or "New Brunswick" or Newfoundland or Labrador or Yukon or Nunavut or "Northwest Territories" or NWT or Alabama or Alaska or Arizona or Arkansas or California or Colorado or Connecticut or Delaware or Florida or Georgia or Hawaii or Idaho or Illinois or Indiana or lowa or Kansas or Kentucky or Louisiana or Maine or Maryland or Massachusetts or Michigan or Minnesota or Mississippi or Missouri or Montana or Nebraska or Nevada or "New Hampshire" or "New Jersey" or "New Mexico" or "New York" or "North Carolina" or "North
Appendix 1 (Continued)

Search String

Search Limiters

Dakota" or Ohio or Oklahoma or

Oregon or Pennsylvania or "Rhode

Island" or "South Carolina" or

"South Dakota" or Tennessee or

Texas or Utah or Vermont or

Virginia or Washington or "West

Virginia" or Wisconsin or Wyoming

or Aguascalientes or "Baja

California" or Campeche or Chiapas

or Chihuahua or Coahuila or

Colima or Durango or Guanajuato

or Guerrero or Hidalgo or Jalisco or

Mexico or Michoacan or Morelos or

Nayarit or "Nuevo Leon" or Oaxaca

or Puebla or Queretaro or

"Quintana Roo" or "San Luis Potosi" or Sinaloa or Sonora or Tabasco or Tamaulipas or Tlaxcala or Veracruz or Yucatan or Zacatecas).mp. [mp = title, abstract, original title, name of substance word, subject heading word, floating sub-heading word, keyword heading word, organism supplementary concept word, protocol supplementary concept word, rare disease supplementary concept word, unique identifier, synonyms] AND ("climate change" or weather or "atmospheric pressure" or "climatic change" or "global warming" or "environmental change" or "climate disaster" or "greenhouse effect" or "climate variability" or "climatic variability" or "carbon emission" or cold or cool or cooling or heat or humid* or ice or precipitation or rain* or season* or snow* or storm or temperature or warm or warming or wind or "ultraviolet radiation" or UV).mp. $[\mathrm{mp}=$ title, abstract, original title, name of substance word, subject heading word, floating subheading word, keyword heading word, organism supplementary concept word, protocol supplementary concept word, rare disease supplementary concept word, unique identifier, synonyms] AND (health or disease* or morbidity or mortality or wellbeing or illness* or wellness or infect* or death or injur* or mental* or emotion*).mp. $[\mathrm{mp}=$ title, abstract, original title, name of substance word, subject heading word, floating subheading word, keyword heading word, organism supplementary concept word, protocol supplementary concept word, rare disease supplementary concept word, unique identifier, synonyms]

\section{Appendix 2}

Data extraction form 


\begin{tabular}{|c|c|}
\hline Data extraction category & Information extracted \\
\hline \multicolumn{2}{|l|}{ Type of research } \\
\hline Research Study & $\begin{array}{l}\text { The article describes research with data } \\
\text { collection and analysis. }\end{array}$ \\
\hline $\begin{array}{l}\text { Review using systematic } \\
\text { methods }\end{array}$ & $\begin{array}{l}\text { The article describes secondary research } \\
\text { that clearly reported review methods. }\end{array}$ \\
\hline \multicolumn{2}{|l|}{ Country* } \\
\hline Canada & $\begin{array}{l}\text { The article describes data collected in } \\
\text { Canada. }\end{array}$ \\
\hline United States of America & $\begin{array}{l}\text { The article describes data collected in } \\
\text { the USA. }\end{array}$ \\
\hline Mexico & $\begin{array}{l}\text { The article describes data collected in } \\
\text { Mexico. }\end{array}$ \\
\hline $\begin{array}{l}\text { Non-North American } \\
\text { Regions }\end{array}$ & $\begin{array}{l}\text { The article describes data collected } \\
\text { outside of North America. Note: To be } \\
\text { included, the article also had to include } \\
\text { data collected in Canada, USA, and/or } \\
\text { Mexico. }\end{array}$ \\
\hline
\end{tabular}

Focus of study*

Climate change impacts

Climate change adaptation The article describes research focused

Climate change mitigation The article describes research focused

Weather variables*

Temperature

Precipitation

UV radiation

Other

Climatic hazards*

Heat events

Cold events

\section{Appendix 2 (Continued)} on strategies/actions to deal with climate change impacts (e.g. flood evacuation plans for coastal communities) in the goal, methods, and/or results section of the article. on strategies/actions to prevent climate change (e.g. reducing emissions) in the goal, methods, and/or results section of the article.

\section{Data extraction category}

The article describes research using data on temperature in the goal, methods, and/or results section of the article.

The article describes research using data on precipitation in the goal, methods, and/or results section of the article.

The article describes research using data on UV radiation in the goal, methods, and/or results section of the article.

The article describes research using data on other climatic variables (e.g. humidity (includes relative humidity or index humidity), seasons, seasonality, changes in season, wind, El Nino/El Nina, etc. in the goal, methods, and/or results section of the article.

The article describes research focused on heat events, including extreme heat, heat waves, in the goal, methods, and/ or results section of the article.

The article describes research focused on cold events, including extreme cold, blizzards, and winter storms, in the goal,
Air quality

Drought

Flooding

Wildfires

Hurricanes

Wildlife changes

Vegetation changes

Freshwater

Ocean conditions

\section{Information extracted}

methods, and/or results section of the article.

The article describes research focused on air quality, including pollution, data on PPM, and greenhouse gas emissions, in the goal, methods, and/or results section of the article.

The article describes research focused on droughts in the goal, methods, and/ or results section of the article.

The article describes research focused on flooding in the goal, methods, and/ or results section of the article.

The article describes research focused on forest and/or wildfires in the goal, methods, and/or results section of the article.

The article describes research focused on hurricanes in the goal, methods, and/or results section of the article.

The article describes research focused on wildlife changes, including changes in vectors (e.g. ticks, mosquitoes), in the goal, methods, and/or results section of the article.

The article describes research focused on vegetation changes, including pollen changes, in the goal, methods, and/or results section of the article.

The article describes research focused on freshwater, including lake/river bodies and drinking water, in the goal, methods, and/or results section of the article.

The article describes research focused on ocean/sea conditions, including sea level rise, and ocean acidity/salinity/ temperature, in the goal, methods, and/ or results section of the article.

Ice extent/stability/duration The article describes research focused on changes in ice extent/stability/ duration, including sea ice and freshwater ice, in the goal, methods, and/or results section of the article.

Coastal erosion

The article describes research focused on coastal erosion in the goal, methods, and/or results section of the article.

Permafrost changes

The article describes research focused on permafrost changes in the goal, methods, and/or results section of the article.

Environmental hazards

The article describes research focused on environmental hazards occurring due to climatic hazards, including reduced crop production, sewage exposure, and fecal runoff, in the goal, methods, and/or results section of the article. 
Appendix 2 (Continued)

Data extraction category Information extracted

\begin{tabular}{ll}
\hline Heat stress, morbidity, and/ & The article describes research focused \\
or mortality & on heat stress, morbidity, and/or \\
& mortality, including heat stroke and \\
& heat stress, in the goal, methods, and/or \\
& results section of the article.
\end{tabular}

Respiratory outcomes

Cardiovascular outcomes

Urinary outcomes

Dermatologic concerns

Fetal health, birth outcomes, and/or maternal health

Cold exposure

Allergies

Nutrition

Waterborne disease

Foodborne disease

Vectorborne disease

Injuries
The article describes research focused on respiratory health, including asthma and COPD, in the goal, methods, and/or results section of the article.

The article describes research focused on cardiovascular disease, including heart attacks and stroke, in the goal, methods, and/or results section of the article.

The article describes research focused on urinary diseases, including urinary tract infections and renal failure, in the goal, methods, and/or results section of the article.

The article describes research focused on dermatologic concerns, including sunburns and melanoma, in the goal, methods, and/or results section of the article.

The article describes research focused on mental health and wellbeing, including suicide, psychoses, and emotional health, in the goal, methods, and/or results section of the article.

The article describes research focused on fetal health, birth outcomes, and/or maternal health in the goal, methods, and/or results section of the article.

The article describes research focused on cold exposures, including frostbite and cold-related morbidity/mortality, in the goal, methods, and/or results section of the article.

The article describes research focused on allergies in the goal, methods, and/ or results section of the article.

The article describes research focused on nutrition, including food security, nutrition transition, and nutrient deficiency, in the goal, methods, and/or results section of the article.

The article describes research focused on waterborne disease in the goal, methods, and/or results section of the article.

The article describes research focused on foodborne disease in the goal, methods, and/or results section of the article.

The article describes research focused on vectorborne disease in the goal, methods, and/or results section of the article.

The article describes research focused on injuries, including fractures and accidents, in the goal, methods, and/or
Appendix 2 (Continued)

\section{Data extraction category Information extracted}

General morbidity and/or
mortality

results section of the article.

mortality

Other outcomes

The article describes research focused on general morbidity and/or mortality that generally references human health in the goal, methods, and/or results section of the article.

The article describes research focused on other health outcomes, including diabetes and electrolyte imbalance, in the goal, methods, and/or results section of the article.

Social variables*

Access to healthcare

The article describes research using data about access to healthcare in the goal, methods, and/or results section of the article.

Sex and/or gender

The article describes research using data on gender and/or sex in the goal, methods, and/or results section of the article.

Age

Income

Livelihood

Ethnicity

Culture

Indigenous Peoples

Rural communities

The article describes research using data on age in the goal, methods, and/or results section of the article.

The article describes research using data on income in the goal, methods, and/or results section of the article.

The article describes research using data on livelihoods, including employment and occupation, in the goal, methods, and/or results section of the article.

The article describes research using data on ethnicity and/or race in the goal, methods, and/or results section of the article.

The article describes research using data on culture in the goal, methods, and/or results section of the article.

The article describes research focused on Indigenous Peoples in the goal, methods, and/or results section of the article.

The article describes research explicitly focused on rural/remote communities in the goal, methods, and/or results section of the article. The authors had to describe the location as "rural", "remote", or other similar terms to fit this classification.

Urban communities The article describes research explicitly focused on urban communities, including cities and metropolitan areas, in the goal, methods, and/or results section of the article. The authors had to describe the location as "urban", "city", "metropolitan", or similar terms to fit this classification.

Coastal communities The article describes research focused on coastal communities in the goal, methods, and/or results section of the article. These communities could also 


\section{Appendix 2 (Continued)}

\begin{tabular}{ll}
\hline Data extraction category & Information extracted \\
\hline & be defined as urban or rural/remote. \\
& The authors had to explicitly describe \\
& the location as "coastal" or similar terms \\
& to fit this classification. \\
& The article describes research using data \\
& on location of residence, including \\
& zipcode/postal code, and \\
neighbourhood, in the goal, methods, & and/or results section of the article. \\
& The article describes research using data \\
& on level of education in the goal, \\
& methods, and/or results section of the \\
article. & \\
Education & The article describes research using data \\
& on housing, including size, age, number \\
of windows, and air conditioning, in the & goal, methods, and/or results section of \\
the article.
\end{tabular}

Future projections*

Qualitative projections

Quantitative projections

No future projections or scenarios

$\begin{array}{ll}\begin{array}{l}\text { No future projections or } \\ \text { scenarios }\end{array} & \begin{array}{l}\text { The article does not describe research } \\ \text { that used projections or future scenarios } \\ \text { in the goal, methods, and/or results } \\ \text { section of the article. }\end{array}\end{array}$

*Categories were not mutually exclusive; that is, more than one category could be selected

\section{Supplementary Information}

The online version contains supplementary material available at https:/doi. org/10.1186/s13643-020-01543-y.

\section{Additional file 1.}

\section{Abbreviations}

CERQual: Confidence in the Evidence from Reviews of Qualitative Research; IPCC: Intergovernmental Panel on Climate Change; MMAT: Mixed Methods Appraisal Tool; PPM: Parts per million; PRISMA: Preferred Reporting Items for Systematic review and Meta-Analyses; PRISMA-P: Preferred Reporting Items for Systematic review and Meta-Analyses, Protocol Extension; USA: United States of America; UV: Ultraviolet

\section{Acknowledgements}

We would like to thank Maria Tan at the University of Alberta Library for the advice, expertise and guidance provided in developing the search strategy for this protocol. Special thanks to those who assisted with methodology refinement, including Etienne de Jongh, Katharine Neale, and Tianna Rusnak.

\section{Authors' contributions}

$\mathrm{SLH}, \mathrm{AC}$, and MDA contributed to the conceptualization, methodology, writing, and editing of the manuscript. $A B$ contributed to the methodology, writing, and editing of the manuscript. SC contributed to the writing and editing of the manuscript. CJW contributed to visualization, writing, and editing of the manuscript. The authors have read and approved the final manuscript.

\section{Funding}

Funding was provided by the Canadian Institutes for Health Research (to $\mathrm{SLH}$ and $\mathrm{AC}$ ). The funding body had no role in the design of the study and collection, analysis, and interpretation of data and in writing the manuscript.

\section{Availability of data and materials \\ Not applicable.}

Ethics approval and consent to participate

Not applicable.

\section{Consent for publication}

Not applicable.

\section{Competing interests}

The authors declare that they have no competing interests.

\section{Author details}

${ }^{1}$ School of Public Health, University of Alberta, ECHA, 1140587 Ave NW, Edmonton, AB T6G 1C9, Canada. ${ }^{2}$ School of Arctic \& Subarctic Studies, Labrador Institute of Memorial University, 219 Hamilton River Road, Stn B, PO Box 490, Happy Valley-Goose Bay, NL AOP 1E0, Canada. ${ }^{3}$ Unidad de Ciencias Sociales, Universidad Autónoma de Yucatán, Calle 61 x 66 \# 525. Col. Centro, Mérida, Yucatán, México.

Received: 30 October 2020 Accepted: 23 November 2020

Published online: 04 January 2021

\section{References}

1. Smith KR, Woodward A, Campbell-Lendrum D, Chadee DD, Honda Y, Liu Q, et al. Human health: impacts, adaptation, and co-benefits. In: Field CB, Barros VR, Dokken DJ, Mach KJ, Mastrandrea MD, Bilir TE, et al., editors. Climate Change 2014: impacts, adaptation, and vulnerability part A: global and sectoral aspects contribution of Working Group II to the Fifth Assessment Report of the Intergovernmental Panel on Climate Change. Cambridge, UK and New York, USA: Cambridge University Press; 2014. p. 709-54.

2. Watts N, Amann M, Ayeb-Karlsson S, Belesova K, Bouley T, Boykoff M, et al. The Lancet Countdown on health and climate change: from 25 years of inaction to a global transformation for public health. Lancet. 2018; 391(10120):581-630.

3. Son JY, Liu JC, Bell ML. Temperature-related mortality: a systematic review and investigation of effect modifiers. Environ Res Lett. 2019;14:073004.

4. Campbell S, Remenyi TA, White CJ, Johnston FH. Heatwave and health impact research: a global review. Heal Place. 2018;53:210-8.

5. Sanderson M, Arbuthnott K, Kovats S, Hajat S, Falloon P. The use of climate information to estimate future mortality from high ambient temperature: a systematic literature review. PLoS One. 2017: e0180369.

6. Rataj E, Kunzweiler K, Garthus-Niegel S. Extreme weather events in developing countries and related injuries and mental health disorders - a systematic review. BMC Public Health. 2016;16:1020.

7. Saulnier DD, Brolin Ribacke K, von Schreeb J. No Calm after the storm: a systematic review of human health following flood and storm disasters. Prehosp Disaster Med. 2017;32(5):568-79.

8. Cunsolo A, Neville E. Ecological grief as a mental health response to climate change-related loss. Nat Clim Chang. 2018;8:275-81.

9. Levy K, Woster AP, Goldstein RS, Carlton EJ. Untangling the impacts of climate change on waterborne diseases: a systematic review of relationships between diarrheal diseases and temperature, rainfall, flooding, and drought. Environ Sci Technol. 2016;50:4905-22.

10. Semenza JC, Herbst S, Rechenburg A, Suk JE, Höser C, Schreiber C, et al. Climate change impact assessment of food- and waterborne diseases. Crit Rev Environ Sci Technol. 2012;42(8):857-90.

11. Cann K, Thomas D, Salmon R, W-J AP, Kay D. Extreme water-related weather events and waterborne disease. Epidemiol Infect. 2013;141:671-86. 
12. Andrade L, O'Dwyer J, O'Neill E, Hynds P. Surface water flooding, groundwater contamination, and enteric disease in developed countries: a scoping review of connections and consequences. Environ Pollut. 2018;236: 540-9.

13. Harper SL, Wright C, Masina S, Coggins S. Climate change, water, and human health research in the Arctic. Water Secur. 2020;10:100062.

14. Park MS, Park KH, Bahk GJ. Interrelationships between multiple climatic factors and incidence of foodborne diseases. Int J Environ Res Public Health. 2018:15:2482.

15. Lake IR, Barker GC. Climate change, foodborne pathogens and illness in higher-income countries. Curr Environ Heal Rep. 2018;5(1):187-96.

16. Lake IR, Gillespie IA, Bentham G, Nichols GL, Lane C, Adak GK, et al. A reevaluation of the impact of temperature and climate change on foodborne illness. Epidemiol Infect. 2009;137(11):1538-47.

17. Lake IR, Hooper L, Abdelhamid A, Bentham G, Boxall AB. a. A, Draper A, et al. Climate change and food security: health impacts in developed countries. Environ Health Perspect. 2012;120(11):1520-6.

18. Springmann M, Mason-D'Croz D, Robinson S, Garnett T, Godfray HCJ, Gollin $D$, et al. Global and regional health effects of future food production under climate change: a modelling study. Lancet. 2016;387(10031):1937-46.

19. Campbell-Lendrum D, Manga L, Bagayoko M, Sommerfeld J. Climate change and vector-borne diseases: what are the implications for public health research and policy? Philos Trans R Soc London. 2015;370:20130552.

20. Sweileh WM. Bibliometric analysis of peer-reviewed literature on climate change and human health with an emphasis on infectious diseases. Glob Health. 2020;16(1):1-17.

21. Ford J. Indigenous health and climate change. Am J Public Health. 2012; 102(7):1260-6.

22. Butler CD. Climate change, health and existential risks to civilization: a comprehensive review (1989-2013). Int J Environ Res Public Health. 2018; 15(10):2266

23. Tong S, Ebi K. Preventing and mitigating health risks of climate change. Environ Res. 2019;174:9-13.

24. Ebi KL, Hess JJ. The past and future in understanding the health risks of and responses to climate variability and change. Int J Biometeorol. 2017;61(S1): $71-80$.

25. Hosking J, Campbell-Lendrum D. How well does climate change and human health research match the demands of policymakers? A scoping review. Environ Health Perspect. 2012;120(8):1076-82.

26. Berry P, Enright PM, Shumake-Guillemot J, Villalobos Prats E, CampbellLendrum D. Assessing health vulnerabilities and adaptation to climate change: a review of international progress. Int J Environ Res Public Health. 2018;15(12):2626.

27. Verner G, Schütte S, Knop J, Sankoh O, Sauerborn R. Health in climate change research from 1990 to 2014: positive trend, but still underperforming. Glob Health Action. 2016;9:30723.

28. Ebi KL, Hasegawa T, Hayes K, Monaghan A, Paz S, Berry P. Health risks of warming of $1.5^{\circ} \mathrm{C}, 2^{\circ} \mathrm{C}$, and higher, above pre-industrial temperatures. Environ Res Lett. 2018;13(6):063007.

29. Bäckstrand K. Civic science for sustainability: reframing the role of experts, policy-makers and citizens in environmental governance. Glob Environ Polit. 2003;3(4):24-41.

30. Susskind L, Jain R, Martyniuk A. Better environmental policy studies: how to design and conduct more effective analyses. Washington, DC: Island Press; 2001. p. 256.

31. Holmes J, Clark R. Enhancing the use of science in environmental policymaking and regulation. Environ Sci Policy. 2008;11(8):702-11.

32. Pearce T, Ford J, Duerden F, Smit B, Andrachuk M, Berrang-Ford $L$, et al. Advancing adaptation planning for climate change in the Inuvialuit Settlement Region (ISR): a review and critique. Reg Environ Chang. 2011;11(1):1-17.

33. Gearheard S, Shirley J. Challenges in community-research relationships: learning from natural science in Nunavut. Arctic. 2007;60(1):62-74.

34. Brownson R, Royer C, Ewing R, McBride T. Researchers and policymakers: travelers in parallel universes. Am J Prev Med. 2006;30(2):164-72.

35. Feldman P. Improving communication between researchers and policy makers in long-term care or, researchers are from Mars; policy makers are from Venus. Gerontologist. 2001;41(3):312-21.

36. Pearce TD, Ford JD, Laidler GJ, Smit B, Duerden F, Allarut M, et al. Community collaboration and climate change research in the Canadian Arctic. Polar Res. 2009:28(1):10-27.
37. Duerden F, Beasley E, Riewe R, Oakes J. Assessing community vulnerabilities to environmental change in the Inuvialuit region. Climate Change: Linking Traditional and Scientific Knowledge. Winnipeg, MB: Aboriginal Issues Press; 2006.

38. Mulrow CD. Rationale for systematic reviews. BMJ. 1994;309(6954):597-9.

39. Tricco A, Lillie E, Zarin W, O'Brien KK, Colquhoun H, Levac D. PRISMA extension for scoping reviews (PRISMA-SCR): checklist and explanation. Ann Intern Med. 2018;169:467-73.

40. Moher D, Liberati A, Tetzlaff J, Altman D. The Prisma Group. Preferred reporting items for systematic reviews and meta-analyses: the PRISMA statement. Ann Intern Med. 2009;151(4):264-9.

41. IPCC. In: Stocker TF, Qin D, Plattner G-K, Tignor M, Allen SK, Boschung J, et al., editors. Climate Change 2013: The Physical Science Basis. Contribution of Working Group I to the Fifth Assessment Report of the Intergovernmental Panel on Climate Change. Cambridge: Cambridge University Press; 2013. 1535 pp

42. IPCC. Managing the risks of extreme events and disasters to advance climate change adaptation. A special report of Working Groups I and II of the Intergovernmental Panel on Climate Change. In: Field CB, Barros V, Stocker TF, Qin D, Dokken DJ, Ebi KL, et al., editors. Cambridge: Cambridge University Press; 2012. p. 582

43. Woodward A, Smith KR, Campbell-Lendrum D, Chadee DD, Honda Y, Liu O et al. Climate change and health: on the latest IPCC report. Lancet. 2014; 383(9924):1185-9.

44. Confalonieri U, Menne B, Akhtar R, Ebi KL, Hauengue M, Kovats RS, et al. Human Health. In: Parry ML, Canziani OF, Palutikof JP, van der Linden PJ, Hanson CE, editors. Climate change 2007: impacts, adaptation and vulnerability contribution of Working Group II to the Fourth Assessment Report of the Intergovernmental Panel on Climate Change. Cambridge: Cambridge University Press; 2007. p. 391-431.

45. IPCC. Climate Change 2014: Impacts, adaptation, and vulnerability. Part B: regional aspects. Contribution of Working Group II to the Fifth Assessment Report of the Intergovernmental Panel on Climate Change. In: Barros VR, Field CB, Dokken DJ, Mastrandrea MD, Mach KJ, Bilir TE, et al., editors. Climate change 2014 impacts, adaptation and vulnerability: part A: global and sectoral aspects. Cambridge, UK and New York, USA: Cambridge University Press; 2014. p. 688.

46. Hong Q, Pluye P, Fàbregues S, Bartlett G, Boardman F, Cargo M, et al. Mixed Methods Appraisal Tool (MMAT), Version 2018. Registration of copyright (\#1148552), Canadian Intellectual Property Office, Industry Canada; 2018. p. $1-11$.

47. Lewin S, Glenton C, Munthe-Kaas H, Carlsen B, Colvin CJ, Gülmezoglu M, et al. Using qualitative evidence in decision making for health and social interventions: an approach to assess confidence in findings from qualitative evidence syntheses (GRADE-CERQual). PLoS Med. 2015;12(10):1001895

\section{Publisher's Note}

Springer Nature remains neutral with regard to jurisdictional claims in published maps and institutional affiliations.

Ready to submit your research? Choose BMC and benefit from:

- fast, convenient online submission

- thorough peer review by experienced researchers in your field

- rapid publication on acceptance

- support for research data, including large and complex data types

- gold Open Access which fosters wider collaboration and increased citations

- maximum visibility for your research: over $100 \mathrm{M}$ website views per year

At $\mathrm{BMC}$, research is always in progress.

Learn more biomedcentral.com/submission 